\title{
On possibilities for studying the problem of human society's evolution using simple mathematical models
}

\author{
L.G. Teklina ${ }^{1}$ \\ ${ }^{1}$ Lobachevsky State University of Nizhny Novgorod, Gagarin Ave. 23, 603950,Nizhny Novgorod, Russia
}

\begin{abstract}
The possibilities for transition from an unstable community with periodical crisis phenomena to a globally steady, stable and dynamically developing community in the situation of technical progress are discussed on the base of a simple mathematical model for an isolated "producers-managers-product" community. The essential tool for studying the mathematical model is pattern recognition methods.
\end{abstract}

Keywords: mathematical model; dynamic system; phase and parametric portraits; pattern recognition

\section{Introduction}

From our point of view, the willingness to study the evolution of the human society more thoroughly may be implemented by creating and investigating mathematical models capable of explaining the observed reality and suggest possible ways to improve it. Constructing an adequate model for such a sophisticated and diverse object as a human society is the task unlikely to be fulfilled. Instead, simple mathematical models are of interest that can open an opportunity to analyze quite complex objects. At the same time, the evolution of the society can hardly be described as a dynamic system. It is possible to introduce major features of the society and describe their interaction using a dynamic system, completing the model with a significant number of parameters characterizing the society under consideration. This way there will be a possibility to study the evolution of the society depending on the model parameters. A sample of this model is a simple mathematical model of the "producersmanagers-product" community given in [1]. A simplified version of this model was analyzed analytically, partially confirmed, and partially supplemented by a small numerical study, whose possibilities turned out to be rather limited for a system with 15 parameters. However, even the incomplete results obtained in this process turned out to be quite interesting. They were analyzed by an historian, confirmed by the facts from the history of global community development and encouraged vivid feedback from the readers [2]. Owing to our attention to this topic and because of the new opportunities for the numerical studies of multidimensional dynamic systems with a large number of parameters we decided to return to this model, but in its full original version.

\section{Brief presentation of the model}

In the study of the model of an isolated "producers (those who actually make the product) - managers (do not make the product but assist in its production) - product (everything necessary for human life, what people consume and use)" community, the values of $x, y, z$ are the numbers of producers, managers and products accumulated by the community. The interaction between them is described (roughly and approximately) in the following simple model as a system of three differential equations:

$$
\begin{aligned}
& x=(a-b x-l y+c z) x \\
& y=(-d-m x-e y+f z) y \\
& z=\left\{\begin{array}{ccc}
F=g \frac{1+\varepsilon_{1} y}{1+\varepsilon_{2} y} \frac{\mu x}{1+\delta z}-h x-k y & \text { if } \quad z>0 \\
0 & \text { if } z=0 \& F \leq 0
\end{array} \text { or } z=0 \& F .0\right.
\end{aligned}
$$

This model reflects the fact that competing people join in the community for more efficient production of the vital product. The model includes 15 parameters that reflect the level of technology development $(g)$, the level of production management $\left(\varepsilon_{1}, \varepsilon_{2}\right)$, the features that take into account an increase in production complexity along with its volume growth and depreciation $(\mu, \delta)$, redistribution of the product between producers and managers $(c, f, h, k)$, competition inside each group ( $a, b$ and $d, e)$, the impact of one group on the other $(l, m)$. The detailed description of the model can be found in [1].

\section{Application of pattern recognition methods to numerical studies of dynamic systems}

A new technique of numerically studying dynamic systems by pattern recognition methods with an active experiment is represented in $[3,4]$. This technique is based on forming selected data on the phenomenon in question using an appropriate mathematical model followed by its pattern recognition analysis. Standard procedures of the technique include the following:

- studying all possible kinds of steady motions in the system phase space (attractors);

- constructing rough phase portraits as the total of attractors and the domains of their attraction in the phase space under given parameter values; 
- studying the dependence of the rough phase portrait on parameter values by constructing a rough parametric portrait of the dynamic system.

These problems are solved for any mathematical models described by the systems of ordinary differential equations regardless of their specific content. Their solution is formal and partially automated. All the obtained results are statistically reliable with the given probability $p_{0}$. In addition, they may provide the basis for solving non-standard problems that are specific for each mathematical model considered. These problems are normally related to studying the dependence of motions in the system phase space on the model parameters. The algorithm for solving them includes the following stages:

- The formulation of the problem as a task for the analysis and research into the dynamics of attractors or system phase portraits.

- The statement of the problem as a pattern recognition task.

- Forming a learning sample to solve the task in the space of the system parameters based on the data on attractors or system phase portraits.

- The selection of the informative features for solving the problem. Informative features for recognition are those system parameters whose change leads to the transition of an object from one recognizable class to another.

- The search of hidden regularities by using different data mining methods on the set of selected features (constructing decision rules of recognition, cluster and regression analysis etc.).

\section{Results of analytical and numerical studies of the mathematical model by pattern recognition methods}

A qualitative study of the model as a dynamic system can be defined as the study of the system phase portraits and their dependence on parameters. Finding all possible types of attractors and system phase portraits in their rough version, constructing a rough parametric portrait of the system was fulfilled by numerical methods based on the use of the ideas and algorithms of pattern recognition. All the results are statistically reliable with the probability $p>0.99$. Below are listed some already published and new data on the qualitative study of the model $[1,4,5]$ that are required for a better understanding of the issue.

Attractors, or steady motions in the system phase space under the given parameter values, correspond to the possible steady communities. The system attractors include the equilibrium states (steady and stable communities) and periodic motions (steady but unstable communities). Only three kinds of possible equilibrium states $\left(x^{*}, y^{*}, z^{*}\right)$ were found: a stable community "producers-managers-product" $P\left(x^{*} \neq 0, y^{*} \neq 0, z^{*} \neq 0\right)$, a stable community "producers-product" $P_{y}\left(x^{*} \neq 0, y^{*}=0, z^{*} \neq 0\right)$ and a stable community "producers" $P_{y z}\left(x^{*} \neq 0, y^{*}=0, z^{*}=0\right)$. A more extensive presence was noted of steady periodic motions, when all the three variables periodically change within the range of $x_{\min } \leq x \leq x_{\max }, y_{\min } \leq y \leq y_{\max }, z_{\min } \leq z \leq z_{\max }$, but more often the following three types of cycles are encountered: $C\left(x_{\min }>0, y_{\min }>0, z_{\min }>0\right)$, $C_{z}\left(x_{\min }>0, y_{\min }>0, z_{\min }=0\right)$ и $C_{y z}\left(x_{\min }>0, y_{\min }=0, z_{\min }=0\right)$. Some cycles are characterized by the periods when some variables stay nearly unchanged. In particular, the moments of achieving and maintaining $z_{\min }=0$ can be considered as crisis phenomena.

Rough phase portraits of the dynamic system give us an insight into how stable communities may exist in the society characterized by a specific set of parameters. Our research has shown that the system may include phase portraits with one attractor, these include three types of equilibrium states $P_{y z}, P_{y}, P$ and all possible cycles, as well as portraits with two attractors: $P_{y z} \& P, P_{y z} \& C, P_{y} \& P, P_{y} \& C$. What are the conditions for these or those phase portraits?

The refined statistically reliable data on the correlation between parameters for different phase portraits are given in tables 1 2, where $g_{0}=\frac{h}{\mu}\left(1+\delta \frac{a m+b d}{b f-c m}\right), \varepsilon=\frac{\varepsilon_{1}}{\varepsilon_{2}}$.

Table 1. Phase portraits under $b f-c m \leq 0$
$b f-c m \leq 0$
\begin{tabular}{ccc} 
Parameters correlation & $g \leq h$ & $g>h$ \\
\hline$c e-l f<0$ & $P_{y z}$ & $\frac{P_{y}, P, C, C_{z}, C_{y z}, \cdots}{P_{y}}$
\end{tabular}

Table 2. Phase portraits under $b f-c m>0$.

\begin{tabular}{cccc}
\multicolumn{4}{c}{$b f-c m>0$} \\
\hline Parameters correlation & $g \leq h$ & $h<g \leq g_{0}$ & $g>g_{0}$ \\
\hline$c e-l f<0$ & $P_{y z}, P_{y z} \& P, P_{y z} \& C$ & $P_{y}, P_{y} \& P_{y} P_{y} \& C$ & $P, C_{z}, C_{y z}, \ldots$ \\
\hline$c e-l f \geq 0$ & $P_{y z}, P_{y z} \& P$ & $P_{y}, P_{y} \& P$ & $P$
\end{tabular}

The obtained results indicate that the following conclusions can be considered statistically reliable:

Conclusion 1. $b f-c m>0 \& \varepsilon>1$ are the necessary conditions for the emergence of steady communities $P$ and $C$ when $g \leq h$. 
Conclusion 2. ce-lf $<0$ \& $\varepsilon>\varepsilon^{*}(g)$ are the necessary conditions for the emergence of unstable communities $C, C_{z}, C_{y z}, \ldots$

Conclusion 3. $g>g^{*}>\frac{h}{\mu}\left(1+\delta \frac{a m+b d}{b f-c m}\right) \& b f-c m>0 \& c e-l f \geq 0$ are the sufficient conditions for the existence of globally steady and stable community $P$ “producers-managers-product".

\section{Problem statement}

The research into the possible ways of the community evolution under certain conditions comes down to the study of the dependence of attractors and phase portraits on parameters. It is worth considering the possibility of moving from an unstable community with periodic crises to the globally steady, stable and dynamically developing community "producers-managersproduct" in the conditions of technical progress. In terms of mathematics, this task can be formulated as follows: which parameters and how should be changed in order to go over from the steady cycle $C\left(C_{z}, C_{y z}, \ldots\right)$ to the globally steady and stable equilibrium $P\left(x^{*} \neq 0, y^{*} \neq 0, z^{*} \neq 0\right)$ which is characterized by the fact that the value $\frac{z^{*}}{x^{*}+y^{*}}$ increases along with the growth of $g$.

Based on the data of the analytical and numerical study shown in tables 1-2 and on the condition of the problem $g \rightarrow \infty$ (technical progress), we can assume that there is a globally steady cycle of any kind $C, C_{z}, C_{y z}, \ldots$, whereas the initial conditions in the phase space may vary. This cycle may exist only provided $c e-l f<0$, but $b f-c m$ may have any values.

\section{On the transition from an unstable community "producers-managers-product" to a stable one}

Is it possible to move to the stable community provided $g \rightarrow \infty$ without changing any other system parameters? Regardless of the $b f-c m$ sign, the specific feature of the globally steady periodic motion (an unstable community) is the fact that with the growth of $g$ while preserving other parameters instability does not disappear, i.e. the cycle never goes into a stable state of equilibrium. The level of the production power development does not ensure stability in the society. Moreover, the growth of $g$ is always accompanied by the growing fluctuations and may cause crisis phenomena: the transition of cycle $C$ to cycles $C_{z}, C_{y z}, \ldots$. An example of this process is given in figure 1 where the graph of dependence $z(t)$ shows the emergence of the time interval when $z=0$ as the parameter $g$ increases. In the presence of crisis phenomena (for example, cycle $C_{z}$ ) the growth of $g$ does not lead to their disappearance either. Furthermore, the crisis phenomena may worsen, e.g., the duration of $z=0$ period may be extended as it occurred in the case shown in figure 2. It is curious that similar effects can be also observed when changing the parameters characterizing the level of production management: when $\varepsilon=\frac{\varepsilon_{1}}{\varepsilon_{2}}$ increases, crisis phenomena do not disappear (figure 2). They may even emerge (figure 1), but on the whole the picture is rather complicated: the period of fluctuations becomes longer, as well as the duration of an interval between crises. At times, crisis periods get shorter (but do not disappear), however at the same time crisis phenomena ( $z$ decline, changes in the population of community groups) are even more pronounced.

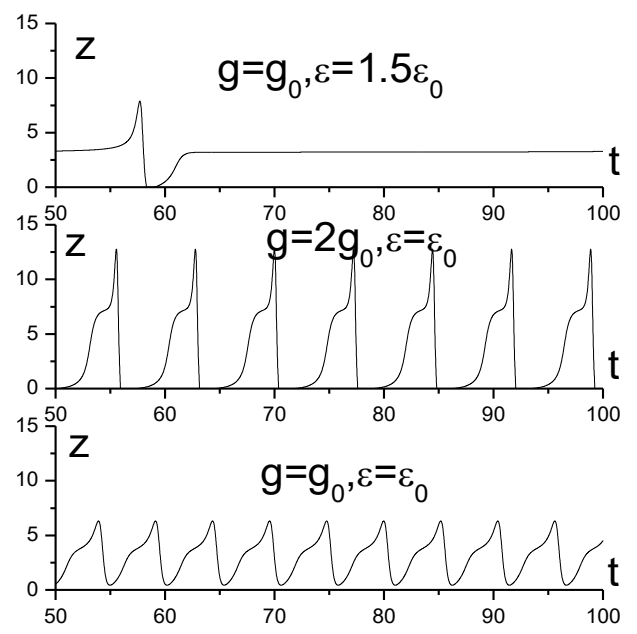

Fig. 1. Changes in $C$ cycle along with growing $g$ and $\varepsilon$.

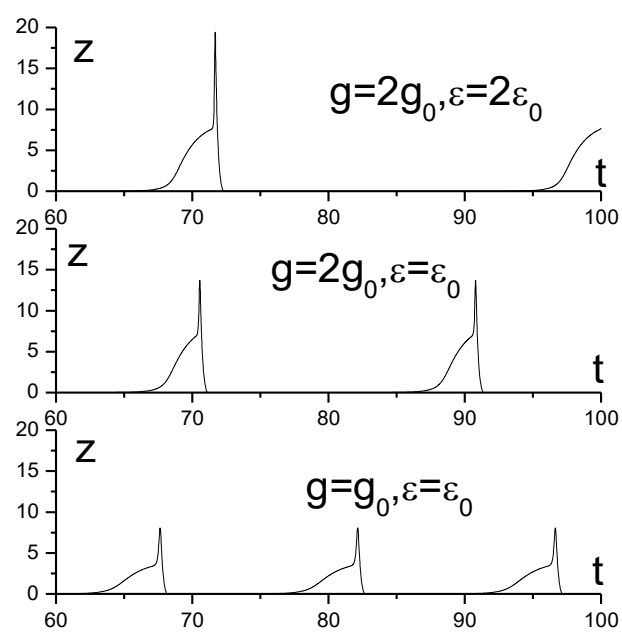

Fig. 2. Changes in $C_{z}$ cycle with growing $g$ and $\varepsilon$.

On retention of all the other parameters and the growth of $g$ the cycle goes to the state of equilibrium only in the case of decreasing $\varepsilon$. At the same time, the higher $g$ is, the lower $\varepsilon$ must be: $\varepsilon<\varepsilon^{*}(g)$, and under $g \rightarrow \infty \varepsilon \rightarrow 0$. Thus, it turns out 
that with $c e-l f<0$ it is necessary to reduce the level of economic management for the transition to a stable community. But what is this stable community? For this equilibrium with the growing $g$ there may be two kinds of changes:

- with growing $g$ (often rather insignificant) the equilibrium transits to the cycle whose changes with the growing $g$ are described above;

- for small $\varepsilon$ the equilibrium $\left(x^{*}, y^{*}, z^{*}\right)$ is preserved, but with the growth of $g$ when $b f-c m \leq 0$ all the coordinates of the equilibrium decrease (and $x^{*}$ decreases faster than the other coordinates), while for $b f-c m>0$ similarly $x^{*} \rightarrow 0$, but $y^{*}$ grows, which, most likely, is the effect of the idealization of the processes described by the model, since this phenomenon will necessarily lead to a change in the parameters of the model, and, consequently, to a change in the phase portrait.

Considering the aforesaid it is possible to conclude that the transition to the stable (non-crisis) community may occur only when changing the parameters characterizing relations between the groups and the redistribution of the products made. In particular, studies have shown (see section 4) that sufficient conditions for emerging and keeping the globally steady equilibrium (stable community of type $\left(x^{*}, y^{*}, z^{*}\right)$ ) are expressed by the two inequalities $b f-c m>0$ and $c e-l f>0$, that can be written in the form $\frac{l}{e}<\frac{c}{f}<\frac{b}{m}$ and must be fulfilled under all $g>g^{*}>\frac{h}{\mu}\left(1+\delta \frac{a m+b d}{b f-c m}\right)$.

\section{On the possibility for the existence of a dynamically developing stable community}

$b f-c m>0 \& c e-l f>0$ are statistically reliable sufficient conditions for the existence of a rough phase portrait with the only steady equilibrium $\left(x^{*}, y^{*}, z^{*}\right)$ whose coordinates increase alongside with the growth of $g$. However, in this case there are two options of the change in the value of $\chi=\frac{z^{*}}{x^{*}+y^{*}}$ : a gradual rise or a very slow fall. What are the conditions for the first version of $\chi$ changes, which might serve as an estimate of the effectiveness of the existing community? To answer this question, we should look at the derivative $\frac{\partial \chi}{\partial g}=\chi_{g}^{\prime}$.

The equilibrium coordinates satisfy the equations

$\left\{\begin{array}{c}a-b x^{*}-l y^{*}+c z^{*}=0 \\ -d-m x^{*}-e y^{*}+f z^{*}=0\end{array}\right.$ wherefrom $x^{*}=\frac{a e+d l}{b e-l m}+\frac{c e-f l}{b e-l m} z^{*}$ and $y^{*}=\frac{a m+b d}{l m-b e}+\frac{c m-b f}{l m-b e}$.

Expression for $\chi$ looks as follows: $\chi=\frac{z^{*}}{x^{*}+y^{*}}=\frac{(b e-l m) z^{*}}{a(e-m)+d(l-b)+(c(e-m)+f(b-l)) z^{*}}$ and, consequently, $\chi_{g}^{\prime}=\frac{(b e-l m)(a(e-m)+d(l-b))\left(z^{*}\right)_{g}^{\prime}}{\left(a(e-m)+d(l-b)+(c(e-m)+f(b-l)) z^{*}\right)^{2}}$.

Provided that $z^{*}$ increases with the growing $g$, i.e. $\left(z^{*}\right)_{g}^{\prime}>0, \chi_{g}^{\prime}>0$ if $(b e-\operatorname{lm})(a(e-m)+d(l-b))>0$.

From the conditions for the existence of the community under consideration, expressed by inequalities $\frac{l}{e}<\frac{c}{f}<\frac{b}{m}$, it follows that $b e-l m>0$, i.e. $\chi_{g}^{\prime}>0$ if $a(e-m)+d(l-b)>0$.

Thus, the conditions for the existence of a globally steady, stable and economically efficient community "producersmanagers-product" may be described with the following inequalities for the parameters describing this community:

1) $g>g^{*}>\frac{h}{\mu}\left(1+\delta \frac{a m+b d}{b f-c m}\right)$

2) $b f-c m>0 \& c e-l f>0$, or $\frac{l}{e}<\frac{c}{f}<\frac{b}{m}$;

3) $g_{t}^{\prime}>0$ - technical progress;

4) $a(e-m)+d(l-b)>0$

Since the above conclusions are based not only on the analytical results, but also on numerical experiments, a control analysis of a large statistical sample (over 30000 cases) was carried out. When conditions (1-2) were met, the rough phase portrait consisted of the only steady equilibrium $\left(x^{*}, y^{*}, z^{*}\right)$ whose coordinates increased when condition (3) was satisfied, and when condition (4) was fulfilled, the value of $\chi=\frac{z^{*}}{x^{*}+y^{*}}$ also increased. 
8. Qualitative description of the inequalities expressing the conditions for the existence of a globally steady, stable and economically efficient community "producers-managers- product"

The simple model in question reflects the essential connections and interactions of the three main elements of the human community: producers- managers- product, but it is very difficult to choose a quantitative equivalent for them. What is there behind the resulting formulas? What features and relations between the groups are reflected by inequalities $(1-4)$ ?

It is evident that inequality (1) requires a certain level of productive forces development, while inequality (3) means the development of the community under technological change. Inequalities (2) and (4) relate the values that reflect production distribution and competition between the groups to the values characterizing relations within each group. These relations should be considered in detail.

Thus, inequality (2) implies that an increase in the share of the product produced for one of the groups (redistribution of products between producers and managers) must be either insignificant or must be accompanied by strong competition within this group and/or a decrease in the pressure on another group.

The inequalities under consideration also impose constraints on the correlation between the values that characterize the competition within the group and the pressure on it, i.e. between $b$ and $l$, between $e$ and $m$.

With $m \geq e$ and $b \geq l \quad a(e-m)+d(l-b) \leq 0$, inequality (4) fails, whereas with $m \geq e$ and $b<l$ inequality (2) fails, because it should be $l<\frac{b e}{m}=b \frac{e}{m} \leq b$. Consequently, inequalities $(2,4)$ require $e>m$, when the competition between managers must be higher than producers' pressure on them.

Suppose $e>m$. If $b \geq l$, inequality (2) is fulfilled by increasing $b(e)$ or decreasing $l(m)$, while inequality (4), which may be recorded as $\frac{b-l}{e-m}<\frac{a}{d}$, by increasing $e$ or reducing $m$, i.e. for the simultaneous achievement of the required correlations it is possible to increase competition between managers. If $b<l$, then inequality (4) $a(e-m)+d(l-b)>0$ is fulfilled, and to fulfil inequality (2) $\frac{l}{e}<\frac{c}{f}<\frac{b}{m}$ it is possible to increase $e$ or decrease $m$. In addition, when $b<l$, inequality (2) imposes the constraint on $l$, namely: $l<b \frac{e}{m}$, i.e. $b<l<b \frac{e}{m}$. Therefore, for producers the correlation between $b$ and $l$ may differ, but at the same time the pressure on producers must be limited, and if the pressure increase is desirable, and sometimes mandatory, it is to be accompanied by an increased competition between managers.

\section{Conclusion}

When discussing the possibilities of studying the objects described by mathematical models, we do not focus on the issue of the adequacy of the models considered (this is to be done by experts from respective fields). Though it should be noted that our research gives additional material for assessing model significance. Nonetheless, the above results, in our opinion, firstly, confirm the wide possibilities of applying new statistical methods in the study of mathematical models, especially those with a large number of parameters, which is a topical problem that can hardly be solved in the general case, and, secondly, the results of this research, without claiming to be complete and comprehensive, allow us to identify significant factors affecting the dynamics of the object under analysis. The data on the community obtained as a result of studying its mathematical model, provide a new knowledge of the possible ways of our society's development and give an insight into the processes taking place in society.

It should be emphasized once again that the results of the research presented in this paper refer to an isolated society and do not take into account any specific features of different communities' interaction. However, even these results are indeed thoughtprovoking.

\section{References}

[1] Neimark YuI. Mathematical Models in Natural Science and Engineering. Springer, 2003; 570 p.

[2] Neimark YuI, Levin AYa. Does God Play Dice? Izvestiya VUZ. Applied nonlinear dynamics 2009; 17(3): 98-136. (in Russian)

[3] Neimark YuI, Kotel'nikov IV, Teklina LG. Coarsened statistical study of applied dynamical systems using pattern recognition methods (part I). Vestnik of Lobachevsky University of Nizhni Novgorod 2012; 5(2); 159-171. (in Russian)

[4] Teklina L, Kotel'nikov I. Analysis and synthesis of dynamic systems using metods of pattern recognition. LAP LAMBERT Academic Publishing, 2015; 129 p. (in Russian)

[5] Neimark YuI, Kotel'nikov IV, Teklina LG. Coarsened statistical study of applied dynamical systems using pattern recognition methods (part II). Vestnik of Lobachevsky University of Nizhni Novgorod 2012; 6(1): 164-174. (in Russian) 